\title{
Delay Feedback Control of the Lorenz-Like System
}

\author{
Qin Chen $(\mathbb{D})$ and Jianguo Gao $\mathbb{D}$ \\ Department of Mathematics and Information Science, North Minzu University, Yinchuan, Ningxia 750021, China
}

Correspondence should be addressed to Jianguo Gao; gaojguo@163.com

Received 18 January 2018; Accepted 7 June 2018; Published 28 June 2018

Academic Editor: Jean Jacques Loiseau

Copyright (C) 2018 Qin Chen and Jianguo Gao. This is an open access article distributed under the Creative Commons Attribution License, which permits unrestricted use, distribution, and reproduction in any medium, provided the original work is properly cited.

\begin{abstract}
We choose the delay as a variable parameter and investigate the Lorentz-like system with delayed feedback by using Hopf bifurcation theory and functional differential equations. The local stability of the positive equilibrium and the existence of Hopf bifurcations are obtained. After that the direction of Hopf bifurcation and stability of periodic solutions bifurcating from equilibrium is determined by using the normal form theory and center manifold theorem. In the end, some numerical simulations are employed to validate the theoretical analysis. The results show that the purpose of controlling chaos can be achieved by adjusting appropriate feedback effect strength and delay parameters. The applied delay feedback control method in this paper is general and can be applied to other nonlinear chaotic systems.
\end{abstract}

\section{Introduction}

Since the discovery of Lorenz chaotic attractor in 1963, chaos has been studied and developed by many scholars, for instance, Rössler system [1], Chua circuit [2], and Chen system [3] et al. However, due to the extremely sensitive characteristics of chaos to the system environment, it is sometimes necessary to suppress or stimulate chaos phenomena in real applications. Therefore, chaos control has attracted more and more attention, and the applications of dynamical systems and chaos involve mathematical biology, financial systems, chemistry, electronic circuits, secure communications, cryptography, and neuroscience research [4-9].

The main goal of chaos control is to eliminate chaotic behavior and stabilize the chaotic system. Especially, when it is useful, we want to generate chaos intentionally. So far, many advanced theories and methodologies have been developed in order to be better in controlling chaos. The existing control method can be classified, mainly, into two categories. The first one, the OGY method [10], which has completely changed the chaos research topic, is based on the invariant manifold structure of unstable orbits. The second one is DFC method, proposed by Pyragas [11, 12], using timedelayed controlling forces. It provides an alternative effective method for feedback control of chaos. In sharp contrast to the formal one, the second is a simple and convenient method of controlling chaos in continuous dynamical system. In order to make further study of the control of chaos via time-delayed feedback, in this paper, we aim to investigate the dynamical behaviors of Lorenz-like system with time delayed.

A new butterfly-shaped Lorenz-like system, which was proposed by Liu [13], is described by the following simple three-dimensional autonomous system with three quadratic terms:

$$
\begin{aligned}
& \dot{x}(t)=a(y(t)-x(t)), \\
& \dot{y}(t)=b x(t)-l x(t) z(t), \\
& \dot{z}(t)=-c z(t)+h x^{2}(t)+k y^{2}(t),
\end{aligned}
$$

where $x, y, z$ are state variables and $a, b, c, l, h, k$ are real valued parameters. When $a=10, b=40, c=2.5, l=1, k=$ $2, h=2$, system (1) admits a chaotic attractor (see Figure 1). The conditions of Hopf bifurcation occurring and the stability and analysis of the equilibrium points have been studied in detail in [14].

The study of [15] shows that the chaotic behavior can be stabilized on various periodic orbits by use of Pyragas timedelayed feedback control. The results of the existence of Hopf bifurcation and effectiveness of delayed feedback have been given [16-23]. Following the idea of Pyragas [12], we add a 

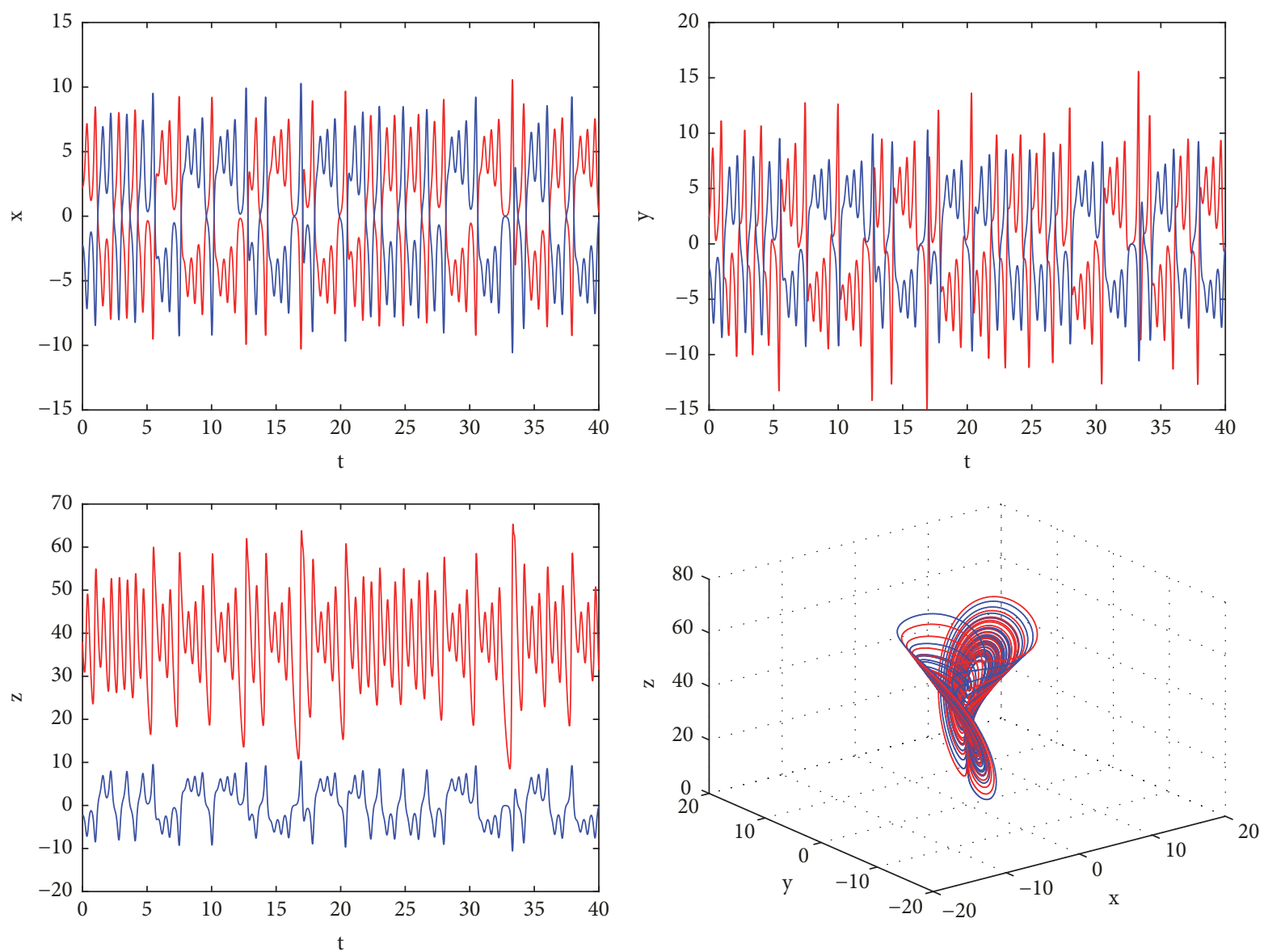

FIGURE 1: System (1) has a chaotic attractor. $a=10, b=40, c=2.5, l=1, k=2$, and $h=2$.

time-delayed force $K(y(t)-y(t-\tau))$ to the second equation of Lorenz-like system (1), and we have

$$
\begin{aligned}
& \dot{x}(t)=a(y(t)-x(t)), \\
& \dot{y}(t)=b x(t)-l x(t) z(t)+K(y(t)-y(t-\tau)), \\
& \dot{z}(t)=-c z(t)+h x^{2}(t)+k y^{2}(t),
\end{aligned}
$$

where $a, b, c, h, k, l>0$. Regarding the time delay $\tau$ as the bifurcation parameter, when $\tau$ passes through some certain critical values, the equilibrium will lose its stability and Hopf bifurcation will take place. By tuning the feedback effect strength $K$, we can implement the control of chaos phenomena of system.

The remainder of this paper is organized as follows. In Section 2, the local stability and the existence of Hopf bifurcation of the control system (2) are determined. In Section 3, some explicit formulas determining the direction and stability of periodic solutions bifurcating from Hopf bifurcations points are demonstrated by applying the normal form theory and the center manifold theorem $[24,25]$. In Section 4, The numerical simulations are carried to demonstrate our theorem analysis. Concluding comments are given in Section 5.

\section{Bifurcation Analysis of the Chaotic System}

In this section, we will investigate the effect of delay on the dynamic behavior of system (2). More specifically, we study the local stability and the existential conditions of Hopf bifurcation. Obviously, when $\tau=0$ system (2) becomes the Lorenz-like system (1). System (1) is linearized at the equilibrium $E_{0}(0,0,0)$ to obtain the Jacobian matrix as follows:

$$
J=\left[\begin{array}{ccc}
-a & a & 0 \\
0 & K-K e^{-\lambda \tau} & 0 \\
0 & 0 & -c
\end{array}\right] .
$$

The associated characteristic equation of (3) is given by

$$
(\lambda+a)(\lambda+c)\left(\lambda-K+K e^{-\lambda \tau}\right)=0 .
$$

When $\tau=0$, (4) has three roots

$$
\begin{aligned}
& \lambda_{1}=0, \\
& \lambda_{2}=-a, \\
& \lambda_{3}=-c .
\end{aligned}
$$


Obviously, if $a>0, c>0$, the equilibrium $E_{0}$ is stable. Therefore, when $\tau=0$, system (2) undergoes a pitchfork bifurcation. Else, the equilibrium $E_{0}$ is unstable.

The Lorenz-like system (2) is symmetric about the $z$-axis, so $E_{+}$and $E_{-}$have the same stability. It is sufficient to analyze the stability of $E_{+}$. By linear transformation

$$
\begin{aligned}
& x_{1}=x-x_{0}, \\
& y_{1}=y-y_{0}, \\
& z_{1}=z-z_{0},
\end{aligned}
$$

system (2) will be

$$
\begin{aligned}
\dot{x}_{1}(t)= & a\left(y_{1}-x_{1}\right), \\
\dot{y}_{1}(t)= & b x_{1}-l z_{0} x_{1}-l x_{0} z_{1}-l x_{1} z_{1} \\
& +K\left[y_{1}-y_{1}(t-\tau)\right], \\
\dot{z}_{1}(t)= & -c z_{1}+2 h x_{0} x_{1}+h x_{1}^{2}+2 k y_{0} y_{1}+k y_{1}^{2} .
\end{aligned}
$$

The Jacobian matrix of $(7)$ at the any point $\left(x_{0}, y_{0}\right)$ is written as

$$
J=\left[\begin{array}{ccc}
-a & a & 0 \\
b-l z_{0} & K-K e^{-\lambda \tau} & -l x_{0} \\
2 h x_{0} & 2 k y_{0} & -c
\end{array}\right]
$$

The characteristic equation of (7) is given by

$$
\begin{aligned}
\lambda^{3}+ & (a+c-K) \lambda^{2} \\
& +\left(-a b+a c-a K-c K+a l z_{0}+2 k l x_{0} y_{0}\right) \lambda \\
& -a b c-a c K+a c l z_{0}+2 a k l x_{0} y_{0}+2 a h l x_{0}^{2} \\
& +\left[K \lambda^{2}+(a+c) K \lambda+a c K\right] e^{-\lambda \tau}=0 .
\end{aligned}
$$

Thus, we will study the distribution of the roots of the third degree exponential polynomial equation

$$
\lambda^{3}+a_{2} \lambda^{2}+a_{1} \lambda+a_{0}+\left(b_{2} \lambda^{2}+b_{1} \lambda+b_{0}\right) e^{-\lambda \tau}=0,
$$

where

$$
\begin{aligned}
& a_{2}=a+c-K, \\
& a_{1}=-a b+a c-a K-c K+a l z_{0}+2 k l x_{0} y_{0}, \\
& a_{0}=-a b c-a c K+a c l z_{0}+2 a k l x_{0} y_{0}+2 a h l x_{0}^{2}, \\
& b_{2}=K, \\
& b_{1}=(a+c) K, \\
& b_{0}=a c K .
\end{aligned}
$$

First of all, we will introduce the results of [26].
Lemma 1. Consider the exponential polynomial

$$
\begin{aligned}
P( & \left.\lambda, e^{-\lambda \tau_{1}}, \ldots, e^{-\lambda \tau_{m}}\right) \\
= & \lambda^{n}+p_{1}^{(0)} \lambda^{n-1}+\cdots+p_{n-1}^{(0)} \lambda^{1}+p_{n}^{(0)} \\
& +\left[p_{1}^{(1)} \lambda^{n-1}+\cdots+p_{n-1}^{(1)} \lambda+p_{n}^{(1)}\right] e^{-\lambda \tau_{1}}+\cdots \\
& +\left[p_{1}^{(m)} \lambda^{n-1}+\cdots+p_{n-1}^{(m)} \lambda+p_{n}^{(m)}\right] e^{-\lambda \tau_{m}},
\end{aligned}
$$

where $\tau_{i} \geqslant 0(i=1,2, \ldots, m)$ and $p_{i}^{(j)}(i=1,2, \ldots, n ; j=$ $0,1,2, \ldots, m)$ are constants. As $\left(\tau_{1}, \tau_{2}, \ldots, \tau_{m}\right)$ vary, the sum of the order of the zeros of $P\left(\lambda, e^{-\lambda \tau_{1}}, \ldots, e^{-\lambda \tau_{m}}\right)$ on the open right half plane can change only if a zero appears on or crosses the imaginary axis.

Obviously, if $i \omega(\omega>0)$ is a root of (10) if and only if $\omega$ satisfies

$$
\begin{aligned}
& -i \omega^{3}-a_{2} \omega^{2}+i a_{1} \omega+a_{0} \\
& \quad+\left(-b_{2} \omega^{2}+i b_{1} \omega+b_{0}\right)(\cos (\omega \tau)-i \sin (\omega \tau)) \\
& =0,
\end{aligned}
$$

separating the real and imaginary parts, we have

$$
\begin{aligned}
& a_{2} \omega^{2}-a_{0}=\left(-b_{2} \omega^{2}+b_{0}\right) \cos (\omega \tau)+b_{1} \omega \sin (\omega \tau), \\
& \omega^{3}-a_{1} \omega=b_{1} \omega \cos (\omega \tau)+\left(b_{2} \omega^{2}-b_{0}\right) \sin (\omega \tau),
\end{aligned}
$$

which is equivalent to

$$
\begin{aligned}
\omega^{6}+ & \left(a_{2}^{2}-b_{2}^{2}-2 a_{1}\right) \omega^{4} \\
& +\left(a_{1}^{2}-2 a_{0} a_{2}-b_{1}^{2}+2 b_{0} b_{2}\right) \omega^{2}+a_{0}^{2}-b_{0}^{2}=0 .
\end{aligned}
$$

Let $z=\omega^{2}$ and denote $p=a_{2}^{2}-b_{2}^{2}-2 a_{1}, q=a_{1}^{2}-2 a_{0} a_{2}-b_{1}^{2}+$ $2 b_{0} b_{2}$, and $r=a_{0}^{2}-b_{0}^{2}$, and then (15) becomes

$$
z^{3}+p z^{2}+q z+r=0
$$

From (16), we have

$$
h(z)=z^{3}+p z^{2}+q z+r
$$

and since $\lim _{z \rightarrow \infty} h(z)=\infty$, we conclude that if $r<0$, then (16) has at least one positive root.

From (17) we have

$$
h^{\prime}(z)=3 z^{2}+2 p z+q .
$$

Clearly, if $\Delta=p^{2}-3 q \leqslant 0$, then the function $h(z)$ is monotone increasing in $z \in[0, \infty)$. Thus, when $r \geqslant 0$ and $\Delta \leqslant 0$, (17) has no positive real roots. On the other hand, when $r \geqslant 0$ and $\Delta>0$, then

$$
3 z^{2}+2 p z+q=0
$$


has two real roots

$$
\begin{aligned}
& z_{1}^{*}=\frac{-p+\sqrt{\Delta}}{3}, \\
& z_{2}^{*}=\frac{-p-\sqrt{\Delta}}{3} .
\end{aligned}
$$

We introduce the following results which were proved by [27].

Lemma 2. For the polynomial (16), we have the following results:

(1) If $\Delta=p^{2}-3 q \leqslant 0$ and $r \geqslant 0$, then (16) does not have positive real root.

(2) If $\Delta=p^{2}-3 q>0$ and $r \geqslant 0$, then (16) has positive real roots if and only if $z_{1}^{*}=(-p+\sqrt{\Delta}) / 3>0$ and $h\left(z_{1}^{*}\right) \leqslant 0$.

(3) If $r<0$, then (16) has at least one positive root.

Suppose that (16) has positive roots. Without loss of generality, we assume that it has three positive roots, which are denoted $z_{1}, z_{2}$, and $z_{3}$. Then (15) has three positive roots

$$
\omega_{k}=\sqrt{z_{k}}, \quad k=1,2,3 .
$$

Now from (14), we have

$$
\cos \omega \tau=\frac{b_{1} \omega^{2}\left(\omega^{2}-a_{1}\right)-\left(a_{2} \omega^{2}-a_{0}\right)\left(b_{2} \omega^{2}-b_{0}\right)}{\left(b_{2} \omega^{2}-b_{0}\right)^{2}+b_{1}^{2} \omega^{2}} .
$$

Then we can write

$$
\begin{aligned}
& \tau_{k}^{(j)} \\
& \quad=\frac{1}{\omega_{k}}\left\{\cos ^{-1}\left(\frac{b_{1} \omega_{k}^{2}\left(\omega_{k}^{2}-a_{1}\right)-\left(a_{2} \omega_{k}^{2}-a_{0}\right)\left(b_{2} \omega_{k}^{2}-b_{0}\right)}{\left(b_{2} \omega_{k}^{2}-b_{0}\right)^{2}+b_{1}^{2} \omega_{k}^{2}}\right)\right. \\
& \quad+2 j \pi\},
\end{aligned}
$$

where $k=1,2,3$ and $j=0,1,2 \cdots$. Then $\pm i \omega_{k}$ is a pair of purely imaginary roots of (10) when $\tau=\tau_{(k)}^{j}$. Define

$$
\tau_{0}=\tau_{k_{0}}^{(0)}=\min _{k \in\{1,2,3\}}\left\{\tau_{k}^{(0)}\right\} .
$$

Note that when $\tau=0$, (10) becomes

$$
\lambda^{3}+\left(a_{2}+b_{2}\right) \lambda^{2}+\left(a_{1}+b_{1}\right) \lambda+a_{0}+b_{0}=0,
$$

which is equivalent to

$$
\begin{gathered}
\lambda^{3}+(a+c) \lambda^{2}+\left(-a b+a c+a l z_{0}+2 k l x_{0} y_{0}\right) \lambda \\
-a b c+a c l z_{0}+2 a k l x_{0} y_{0}+2 a h l x_{0}^{2}=0 .
\end{gathered}
$$

Therefore, applying Lemmas 1 and 2 to (10), we obtain the following lemma.

Lemma 3. For (10) we have

(1) If $\Delta=p^{2}-3 q \leqslant 0$ and $r \geqslant 0$, then all roots with positive real parts of (10) have the same sum to those of (24) for all $\tau \geqslant 0$;

(2) If $\Delta=p^{2}-3 q>0, z_{1}^{*}>0, h\left(z_{1}^{*}\right) \leqslant 0$, then all roots with positive real parts of (10) have the same sum to those of (24) for $\tau \in\left[0, \tau_{0}\right)$.
Now let us consider the behavior of the roots of (10) near $\tau=\tau_{k}^{(j)}$. To do this, we assume that $\lambda(\tau)=\alpha(\tau)+i \omega(\tau)$ is a solution of (10) satisfying $\alpha\left(\tau_{k}^{(j)}\right)=0, \omega\left(\tau_{k}^{(j)}\right)=\omega_{k}(k=$ $1,2,3)$. Then we have the following transversality condition.

Lemma 4. If $h^{\prime}\left(z_{k}\right) \neq 0$, then $d \operatorname{Re} \lambda\left(\tau_{k}^{(j)}\right) / d \tau \neq 0, h^{\prime}\left(z_{k}\right)$, and $d \operatorname{Re} \lambda\left(\tau_{k}^{(j)}\right) / d \tau$ have the same sign.

Proof. Substituting $\lambda(\tau)$ into (10) and taking the derivative with respect to $\tau$, we obtain

$$
\begin{aligned}
{\left[\frac{d \lambda}{d \tau}\right]^{-1}=} & \frac{3 \lambda^{2}+2 a_{2} \lambda+a_{1}}{\lambda\left(b_{2} \lambda^{2}+b_{1} \lambda+b_{0}\right) e^{-\lambda \tau}} \\
& +\frac{2 b_{2} \lambda+b_{1}}{\lambda\left(b_{2} \lambda^{2}+b_{1} \lambda+b_{0}\right)}-\frac{\tau}{\lambda}
\end{aligned}
$$

From (14), we obtain

$$
\begin{aligned}
& {\left[\frac{d(\operatorname{Re} \lambda(\tau))}{d \tau}\right]_{\tau=\tau_{k}^{(j)}}^{-1}} \\
& =\operatorname{Re}\left[\frac{3 \lambda^{2}+2 a_{2} \lambda+a_{1}}{\lambda\left(b_{2} \lambda^{2}+b_{1} \lambda+b_{0}\right) e^{-\lambda \tau}}\right]_{\tau=\tau_{k}^{(j)}} \\
& +\operatorname{Re}\left[\frac{2 b_{2} \lambda+b_{1}}{\lambda\left(b_{2} \lambda^{2}+b_{1} \lambda+b_{0}\right)}\right]_{\tau=\tau_{k}^{(j)}} \\
& =\operatorname{Re}\left[\frac { 3 \lambda ^ { 2 } + 2 a _ { 2 } \lambda + a _ { 1 } } { \lambda ( b _ { 2 } \lambda ^ { 2 } + b _ { 1 } \lambda + b _ { 0 } ) } \left(\cos \left(\omega_{k} \tau_{k}^{(j)}\right)\right.\right. \\
& \left.\left.+i \sin \left(\omega_{k} \tau_{k}^{(j)}\right)\right)\right]_{\tau=\tau_{k}^{(j)}} \\
& +\operatorname{Re}\left[\frac{2 b_{2} \lambda+b_{1}}{\lambda\left(b_{2} \lambda^{2}+b_{1} \lambda+b_{0}\right)}\right]_{\tau=\tau_{k}^{(j)}}=\frac{1}{\Lambda}\left[3 \omega_{k}^{6}\right. \\
& +2\left(a_{2}^{2}-b_{2}^{2}-2 a_{1}\right) \omega_{k}^{4}+\left(a_{1}^{2}-2 a_{0} a_{2}-b_{1}^{2}+2 b_{0} b_{2}\right) \\
& \left.\cdot \omega_{k}^{2}\right]=\frac{1}{\Lambda}\left[3 \omega_{k}^{6}+2 p \omega_{k}^{4}+q \omega_{k}^{2}\right]=\frac{z_{k}}{\Lambda}\left(3 z_{k}^{2}+2 p z_{k}\right. \\
& +q)=\frac{z_{k}}{\Lambda} h^{\prime}\left(z_{k}\right) \text {, }
\end{aligned}
$$

where $\Lambda=b_{1}^{2} \omega_{k}^{4}+\left(b_{0} \omega_{k}-b_{2} \omega_{k}^{3}\right)^{2}$, and since $z_{k}>0$, we can conclude that $h^{\prime}(z)$ and $d \operatorname{Re} \lambda\left(\tau_{k}^{(j)}\right) / d \tau$ have the same sign. This completes the proof.

Theorem 5. Suppose $\Delta=p^{2}-3 q>0, z_{1}^{*}>0, h\left(z_{1}^{*}\right)<$ 0 and Routh-Hurwitz criterion for (10) hold and system (2) undergoes Hopf bifurcation at the equilibria $E_{ \pm}$when $\tau=\tau_{k}^{(j)}$ $(k=1,2,3$ and $=0,1,2, \cdots)$. In addition, if $\tau_{1}^{(0)}>\tau_{2}^{(0)}$, then there exists $m \in N$ such that $\tau_{2}^{(0)}<\tau_{1}^{(0)}<\tau_{2}^{(1)}<$ $\tau_{1}^{(1)}<\cdots<\tau_{2}^{(m)}<\tau_{1}^{(m)}<\tau_{2}^{(m+1)}<\tau_{1}^{(m+1)}$, and the equilibria $E_{+}$of system (2) are asymptotically stable for $\tau \in$ $\left[0, \tau_{2}^{(0)}\right] \bigcup\left[\tau_{1}^{(0)}, \tau_{2}^{(1)}\right] \bigcup \cdots \cup\left[\tau_{1}^{(m-1)}, \tau_{2}^{(m)}\right] \bigcup\left[\tau_{1}^{(m)}, \tau_{2}^{(m+1)}\right]$, and 
they are unstable for $\tau \in\left[\tau_{2}^{(0)}, \tau_{1}^{(0)}\right] \bigcup\left[\tau_{2}^{(1)}, \tau_{1}^{(1)}\right] \bigcup \cdots \cup\left[\tau_{2}^{(m)}\right.$, $\left.\tau_{1}^{(m)}\right] \bigcup\left[\tau_{2}^{(m+1)},+\infty\right]$. Furthermore, system (2) undergoes Hopf bifurcation at the equilibria $E_{ \pm}$when $\tau=\tau_{k}^{(j)}(k=1,2,3$ and $=0,1,2, \cdots)$.

\section{Direction and Stability of Hopf Bifurcation Period Solution}

In previous section, we obtained the conditions when the Hopf bifurcation occurs. In this section, we shall study the direction and the stability of the bifurcations with normal form theory and central manifold theorem. During this section we always assume that system (2) undergoes Hopf bifurcation at the equilibrium $E_{+}$for $\tau=\tau_{k}$.

Let $u_{1}=x-x_{0}, u_{2}=y-y_{0}, u_{3}=z-z_{0}$, $\bar{u}_{i}(t)=u_{i}(t \tau)$, and $\tau=\mu+\tau_{k}$, dropping the bars for simplifications of notations. The nonlinear system (2) can be transformed into an functional differential equation (FDE) in $C \in C\left([-1,0], R^{3}\right)$ as

$$
\dot{u}(t)=L_{\mu}\left(u_{t}\right)+f\left(\mu, u_{t}\right) \text {, }
$$

where $u(t)=\left(u_{1}(t), u_{2}(t), u_{3}(t)\right)^{T} \in R^{3}$ and $L_{\mu}: C \longrightarrow R, f$ : $R \times C \longrightarrow R$ are given, respectively, by

$$
\begin{aligned}
L_{\mu}(\varphi)= & \left(\mu+\tau_{k}\right)\left(\begin{array}{ccc}
-a & a & 0 \\
b-l z_{0} & K & -l x_{0} \\
2 h x_{0} & 2 k y_{0} & -c
\end{array}\right)\left(\begin{array}{l}
\varphi_{1}(0) \\
\varphi_{2}(0) \\
\varphi_{3}(0)
\end{array}\right) \\
& +\left(\mu+\tau_{k}\right)\left(\begin{array}{ccc}
0 & 0 & 0 \\
0 & -K & 0 \\
0 & 0 & 0
\end{array}\right)\left(\begin{array}{c}
\varphi_{1}(-1) \\
\varphi_{2}(-1) \\
\varphi_{3}(-1)
\end{array}\right)
\end{aligned}
$$

and

$$
f(\tau, \varphi)=\left(\mu+\tau_{k}\right)\left(\begin{array}{c}
0 \\
-l \varphi_{1}(0) \varphi_{3}(0) \\
h \varphi_{1}^{2}(0)+k \varphi_{2}^{2}(0)
\end{array}\right) .
$$

By the Riesz representation theorem, there exists a function $\eta(\theta, \mu)$ of bounded variation for $\theta \in[-1,0]$, such that

$$
L_{\mu}(\varphi)=\int_{-1}^{0} d \eta(\theta, 0) \varphi(\theta), \quad \theta \in C .
$$

If we choose

$$
\begin{aligned}
\eta(\theta, \mu)= & \left(\mu+\tau_{k}\right)\left(\begin{array}{ccc}
-a & a & 0 \\
b-l z_{0} & K & -l x_{0} \\
2 h x_{0} & 2 k y_{0} & -c
\end{array}\right) \delta(\theta) \\
& -\left(\mu+\tau_{k}\right)\left(\begin{array}{ccc}
0 & 0 & 0 \\
0 & -K & 0 \\
0 & 0 & 0
\end{array}\right) \delta(\theta+1),
\end{aligned}
$$

where $\delta(\cdot)$ is a Dirac delta function, for $\varphi \in C\left([-1,0], R^{3}\right)$, define

$$
A(\mu) \varphi= \begin{cases}\frac{d \varphi(\theta)}{d \theta}, & \theta \in[-1,0), \\ \int_{-1}^{0} d \eta(\mu, s) \varphi(s), & \theta=0\end{cases}
$$

and

$$
R(\mu) \varphi= \begin{cases}0, & \theta \in[-1,0) \\ f(\mu, \varphi), & \theta=0 .\end{cases}
$$

For convenience, we can write system (29) into an operator equation

$$
\dot{u}(t)=A(\mu) u_{t}+R(\mu) u_{t},
$$

where $u_{t}(\theta)=u(t+\theta), \theta \in[-1,0]$.

For $\psi \in C^{1}\left([0,1],\left(R^{3}\right)^{*}\right)$, define

$$
A^{*} \psi(s)= \begin{cases}-\frac{d \psi(s)}{d s}, & s \in(0,1], \\ \int_{-1}^{0} d \eta^{T}(t, 0) \psi(-t), & s=0,\end{cases}
$$

and a bilinear inner product

$$
\begin{aligned}
\langle\psi(s), \varphi(\theta)\rangle= & \bar{\psi}(0) \varphi(0) \\
& -\int_{-1}^{0} \int_{\xi=0}^{\theta} \bar{\psi}(\xi-\theta) d \eta(\theta) \varphi(\xi) d \xi
\end{aligned}
$$

where $\eta(\theta)=\eta(\theta, 0)$. Obviously $A^{*}$ and $A(0)$ are adjoint operators. By the discussion in Section 2, we know that $\pm i \omega_{k} \tau_{k}$ are eigenvalues of $A(0)$. Thus they are also eigenvalues of $A^{*}$. We need to calculate the eigenvectors of $A^{*}$ and $A(0)$ corresponding to $-i \omega_{k} \tau_{k}$ and $i \omega_{k} \tau_{k}$, respectively.

Let $q(\theta)=(1, \alpha, \beta)^{T} e^{i \theta \omega_{k} \tau_{k}}$ be the eigenvector of $\mathrm{A}(0)$, i.e., $A(0) q(\theta)=i \omega_{k} \tau_{k} q(\theta)$ and then we have

$$
\begin{aligned}
& \tau_{k}\left(\begin{array}{ccc}
i \omega_{k}+a & -a & 0 \\
l z_{0}-b & i \omega_{k}-K+K e^{-i \omega_{k} \tau} & l x_{0} \\
-2 h x_{0} & -2 k y_{0} & i \omega_{k}+c
\end{array}\right) q(0) \\
& =\left(\begin{array}{l}
0 \\
0 \\
0
\end{array}\right)
\end{aligned}
$$

Then it is easy to obtain

$$
\begin{aligned}
q(0) & =(1, \alpha, \beta)^{T} \\
& =\left(1, \frac{i \omega_{k}+a}{a}, \frac{2 a h x_{0}+2 k y_{0}\left(i \omega_{k}+a\right)}{a\left(i \omega_{k}+c\right)}\right)^{T} .
\end{aligned}
$$


Similarly, we suppose that $q^{*}(s)=D\left(1, \alpha^{*}, \beta^{*}\right) e^{i s \omega_{k} \tau_{k}}$ is the eigenvector of $A^{*}$ corresponding to $-i \omega_{k} \tau_{k}$. From the definition of $A^{*}$, we have

$$
\begin{aligned}
& q^{*}(s)=D\left(1, \alpha^{*}, \beta^{*}\right) e^{i s \omega_{k} \tau_{k}}=D(1, \\
& \frac{-\left(a-i \omega_{k}\right)\left(c-i \omega_{k}\right)}{\left(b-l z_{0}\right)\left(i \omega_{k}-c\right)+2 h l x_{0}^{2}}, \\
& \left.\frac{\left(a-i \omega_{k}\right) l x_{0}}{\left(b-l z_{0}\right)\left(i \omega_{k}-c\right)+2 h l x_{0}^{2}}\right) e^{i s \omega_{k} \tau_{k}} .
\end{aligned}
$$

Where $D$ is a constant such that $\left\langle q^{*}(s), q(\theta)\right\rangle=1$, by (38) we get

$$
\begin{aligned}
& \left\langle q^{*}(s), q(\theta)\right\rangle=\bar{D}\left(1, \overline{\alpha^{*}}, \overline{\beta^{*}}\right)(1, \alpha, \beta)^{T} \\
& \quad-\int_{-1}^{0} \int_{\xi=0}^{\theta} \bar{D}\left(1, \overline{\alpha^{*}}, \overline{\beta^{*}}\right) e^{-i(\xi-\theta) \omega_{k} \tau_{k}} d \eta(\theta)(1, \alpha, \beta)^{T} \\
& \cdot e^{i \xi \omega_{k} \tau_{k}} d \xi=\bar{D}\left(1+\overline{\alpha^{*}} \alpha+\overline{\beta^{*}} \beta\right) \\
& \quad-\int_{-1}^{0} \bar{D}\left(1, \overline{\alpha^{*}}, \overline{\beta^{*}}\right) \theta e^{i \theta \omega_{k} \tau_{k}} d \eta(\theta)(1, \alpha, \beta)^{T}=\bar{D}(1 \\
& \left.+\overline{\alpha^{*}} \alpha+\overline{\beta^{*}} \beta-K \tau_{k} \overline{\alpha^{*}} \alpha e^{-i \omega_{k} \tau_{k}}\right) .
\end{aligned}
$$

Therefore, we can choose $D$ as

$$
D=\frac{1}{1+\bar{\alpha} \alpha^{*}+\bar{\beta} \beta^{*}-K \tau_{k} \bar{\alpha} \alpha^{*} e^{i \omega_{k} \tau_{k}} .}
$$

In the following, we apply the idea in Hassrd and Kazarinoff and Wan [24] to compute the coordinates describing the center manifold $C_{0}$ at $\mu=0$. Let $u_{t}$ be the solution of (29) when $\mu=0$.

Define

$$
\begin{aligned}
z(t) & =\left\langle q^{*}, u_{t}\right\rangle, \\
W(t, \theta) & =u_{t}(\theta)-2 \operatorname{Re}\{z(t) q(\theta)\} .
\end{aligned}
$$

On the center manifold $C_{0}$, we get

$$
W(t, \theta)=W(z(t), \bar{z}(t), \theta),
$$

where

$$
\begin{aligned}
W(z(t), \bar{z}(t), \theta)= & W_{20}(\theta) \frac{z^{2}}{2}+W_{11}(\theta) z \bar{z} \\
& +W_{02}(\theta) \frac{\bar{z}^{2}}{2}+W_{30}(\theta) \frac{z^{3}}{6} \\
& +\cdots,
\end{aligned}
$$

$z$ and $\bar{z}$ are local coordinates for center manifold $C_{0}$ in the direction of $q^{*}$ and $\bar{q}^{*}$. It is clear that $W$ is real if $u_{t}$ is real. Again, we are only considering real solutions. Therefore, to get the solution of $u_{t} \in C_{0}$ of (29), since $\mu=0$, We have

$$
\begin{gathered}
\dot{z}(t)=i \omega_{k} \tau_{k} z+\left\langle\bar{q}^{*}(\theta) f(0, W(z(t), \bar{z}(t), \theta))\right. \\
+2 \operatorname{Re}\{z(t) q(\theta)\}\rangle=i \omega_{k} \tau_{k} z+\bar{q}^{*}(0) \\
\cdot f(0, W(z(t), \bar{z}(t), 0)+2 \operatorname{Re}\{z(t) q(0)\}) .
\end{gathered}
$$

Let $f(0, W(z(t), \bar{z}(t), 0)+2 \operatorname{Re}\{z(t) q(0)\})=f_{0}(z, \bar{z})$, and then $\dot{z}=i \omega_{k} \tau_{k} z+q^{*}(0) f_{0}(z, \bar{z})$. We write this equation as

$$
\dot{z}(t)=i \omega_{k} \tau_{k} z+g(z, \bar{z}),
$$

where

$$
\begin{aligned}
g(z, \bar{z}) & =\bar{q}^{*}(0) f_{0}(z, \bar{z}) \\
& =g_{20} \frac{z^{2}}{2}+g_{11} z \bar{z}+g_{02} \frac{\bar{z}^{2}}{2}+g_{21} \frac{z^{2} \bar{z}}{2}+\cdots .
\end{aligned}
$$

Since

$$
q(\theta)=(1, \alpha, \beta)^{T} e^{i \theta \omega_{k} \tau_{k}},
$$

and

$$
\begin{aligned}
u_{t}(\theta) & =\left(u_{1 t}(\theta), u_{2 t}(\theta), u_{3 t}(\theta)\right) \\
& =W(t, \theta)+z(t) q(\theta)+\bar{z}(t) \bar{q}(\theta)
\end{aligned}
$$

we have

$$
\begin{aligned}
u_{1 t}(0)= & z+\bar{z}+W_{20}^{(1)}(0) \frac{z^{2}}{2}+W_{11}^{(1)}(0) z \bar{z} \\
& +W_{02}^{(1)}(0) \frac{\bar{z}^{2}}{2}+O\left(\mid(z, \bar{z} \mid)^{3}\right), \\
u_{2 t}(0)= & \alpha z+\bar{\alpha} \bar{z}+W_{20}^{(2)}(0) \frac{z^{2}}{2}+W_{11}^{(2)}(0) z \bar{z} \\
& +W_{02}^{(2)}(0) \frac{\bar{z}^{2}}{2}+O\left(\mid(z, \bar{z} \mid)^{3}\right), \\
u_{3 t}(0)= & \beta z+\bar{\beta} \bar{z}+W_{20}^{(3)}(0) \frac{z^{2}}{2}+W_{11}^{(3)}(0) z \bar{z} \\
& +W_{02}^{(3)}(0) \frac{\bar{z}^{2}}{2}+O\left(\mid(z, \bar{z} \mid)^{3}\right) .
\end{aligned}
$$

In view of (49), we get

$$
\begin{aligned}
& g(z, \bar{z})=\bar{q}^{*}(0) f_{0}(z, \bar{z})=\bar{D} \tau_{k}\left(1, \overline{\alpha^{*}}, \overline{\beta^{*}}\right) \\
& \cdot\left(\begin{array}{c}
0 \\
-l u_{1 t}(0) u_{3 t}(0) \\
h u_{1 t}^{2}(0)+k u_{2 t}^{2}(0)
\end{array}\right)=\bar{D} \tau_{k} \overline{\alpha^{*}}(-l)[(z+\bar{z} \\
& +W_{20}^{(1)}(0) \frac{z^{2}}{2}+W_{11}^{(1)}(0) z \bar{z}+W_{02}^{(1)}(0) \frac{\bar{z}^{2}}{2} \\
& \left.\left.+O\left(\mid(z, \bar{z} \mid)^{3}\right)\right)\right]\left[\beta z+\bar{\beta} \bar{z}+W_{20}^{(3)}(0) \frac{z^{2}}{2}\right. \\
& \left.+W_{11}^{(3)}(0) z \bar{z}+W_{02}^{(3)}(0) \frac{\bar{z}^{2}}{2}+O\left(\mid(z, \bar{z} \mid)^{3}\right)\right]
\end{aligned}
$$




$$
\begin{aligned}
& +\bar{D} \tau_{k} \overline{\beta^{*}} h\left(\left[z+\bar{z}+W_{20}^{(1)}(0) \frac{z^{2}}{2}+W_{11}^{(1)}(0) z \bar{z}\right.\right. \\
& \left.\left.+W_{02}^{(1)}(0) \frac{\bar{z}^{2}}{2}+O\left(\mid(z, \bar{z} \mid)^{3}\right)\right]\right)^{2}+\bar{D} \tau_{k} \overline{\beta^{*} k}([\alpha z \\
& +\bar{\alpha} \bar{z}+W_{20}^{(2)}(0) \frac{z^{2}}{2}+W_{11}^{(2)}(0) z \bar{z}+W_{02}^{(2)}(0) \frac{\bar{z}^{2}}{2} \\
& \left.\left.+O\left(\mid(z, \bar{z} \mid)^{3}\right)\right]\right)^{2}=\bar{D} \tau_{k}\left[M z^{2}+N z \bar{z}+P \bar{z}^{2}\right. \\
& \left.+Q z^{2} \bar{z}+\text { H.O.T }\right] .
\end{aligned}
$$

Comparing the coefficients with (49), we get

$$
\begin{aligned}
M= & -l \overline{\alpha^{*}} \beta+\overline{\beta^{*}}\left(h+k \alpha^{2}\right), \\
N= & -l \overline{\alpha^{*}}(\beta+\bar{\beta})+\overline{\beta^{*}}(2 h+2 k \alpha \bar{\alpha}), \\
P= & -l \bar{\alpha} \bar{\beta}+\overline{\beta^{*}}\left(h+k \bar{\alpha}^{2}\right), \\
Q= & -\overline{\alpha^{*}}\left[W_{11}^{(3)}+\frac{1}{2} W_{20}^{(3)}+\frac{1}{2} W_{20}^{(1)} \bar{\beta}+W_{11}^{(1)} \beta\right] \\
& +\overline{\beta^{*}}\left[2 h W_{11}^{(1)}+h W_{20}^{(1)}+2 k \alpha W_{11}^{(2)}+k \bar{\alpha} W_{20}^{(2)}\right] .
\end{aligned}
$$

where

$$
\begin{aligned}
& g_{20}=2 \bar{D} \tau_{k} M, \\
& g_{11}=\bar{D} \tau_{k} N, \\
& g_{02}=2 \bar{D} \tau_{k} P, \\
& g_{21}=2 \bar{D} \tau_{k} Q .
\end{aligned}
$$

Since $W_{11}(\theta)$ and $W_{20}(\theta)$ in $g_{21}$ are unknown, we still need to compute them. By (36) and (44), we drive

$$
\begin{aligned}
\dot{W} & =\dot{u}_{t}-\dot{z} q-\dot{\bar{z}} \bar{q} \\
& = \begin{cases}A W-2 \operatorname{Re}\left\{\bar{q}^{*}(0) f_{0} q(\theta)\right\}, & \theta \in[-1,0), \\
A W-2 \operatorname{Re}\left\{\bar{q}^{*}(0) f_{0} q(0)\right\}+f_{0}, & \theta=0 .\end{cases}
\end{aligned}
$$

Let

$$
\begin{aligned}
\dot{H} & (z, \bar{z}, \theta) \\
& = \begin{cases}2 \operatorname{Re}\left\{\bar{q}^{*}(0) f_{0} q(\theta)\right\}, & \theta \in[-1,0), \\
2 \operatorname{Re}\left\{\bar{q}^{*}(0) f_{0} q(0)\right\}+f_{0}, & \theta=0 .\end{cases}
\end{aligned}
$$

We can rewrite

$$
\dot{W}=A(0) W+H(z, \bar{z}, \theta),
$$

where

$$
H(z, \bar{z}, \theta)=H_{20}(\theta) \frac{z^{2}}{2}+H_{11}(\theta) z \bar{z}+H_{02}(\theta) \frac{\bar{z}^{2}}{2} \cdots
$$

From (56) and (59) and the definition of $W$, expanding the above series and comparing the coefficients, we get

$$
\begin{aligned}
& \left(A(0)-2 i \omega_{k} \tau_{k}\right) W_{20}(\theta)=-H_{20}(\theta), A(0) W_{11}(\theta) \\
& \quad=-H_{11}(\theta), \cdots .
\end{aligned}
$$

And from (56), we know that, for $\theta \in[-1,0)$,

$$
\begin{aligned}
H(z, \bar{z}, \theta) & =-\bar{q}^{*}(0) f_{0} q(\theta)-q^{*}(0) \bar{f}_{0} \bar{q}(\theta) \\
& =-g(z, \bar{z}) q(\theta)-\bar{g}(z, \bar{z}) \bar{q}(\theta) .
\end{aligned}
$$

Comparing the coefficients with (59), we obtain

$$
\begin{aligned}
& H_{20}(\theta)=-g_{20} q(\theta)-\bar{g}_{02} \bar{q}(\theta), \\
& H_{11}(\theta)=-g_{11} q(\theta)-\bar{g}_{11} \bar{q}(\theta) .
\end{aligned}
$$

By (60), (62), and the definition of $A(0)$, it follows that

$$
\dot{W}_{20}=2 i \omega_{k} \tau_{k} W_{20}(\theta)+g_{20} q(\theta)+\bar{g}_{02} \bar{q}(\theta) .
$$

Substituting $q(\theta)=(1, \alpha, \beta)^{T} e^{i \theta \omega_{k} \tau_{k}}$ into the last equation, we can obtain the solution of it, which reads

$$
\begin{aligned}
W_{20}(\theta)= & \frac{i g_{20}}{\omega_{k} \tau_{k}} q(0) e^{i \theta \omega_{k} \tau_{k}}+\frac{i \bar{g}_{02}}{3 \omega_{k} \tau_{k}} \bar{q}(0) e^{-i \theta \omega_{k} \tau_{k}} \\
& +E_{1} e^{2 i \theta \omega_{k} \tau_{k}},
\end{aligned}
$$

where $E_{1}=\left(E_{1}^{(1)}, E_{1}^{(2)}, E_{1}^{(3)}\right)^{T} \in R^{3}$ is a constant vector.

Similarly, in view of (60), (63), and the definition of $A(0)$, we get

$$
\begin{aligned}
\dot{W}_{11}(\theta)= & g_{11} q(\theta)+\bar{g}_{11} \bar{q}(\theta), \\
W_{11}(\theta)= & -\frac{i g_{11}}{\omega_{k} \tau_{k}} q(0) e^{i \theta \omega_{k} \tau_{k}}+\frac{i \bar{g}_{11}}{\omega_{k} \tau_{k}} \bar{q}(0) e^{-i \theta \omega_{k} \tau_{k}} \\
& +E_{2},
\end{aligned}
$$

where $E_{2}=\left(E_{2}^{(1)}, E_{2}^{(2)}, E_{2}^{(3)}\right)^{T} \in R^{3}$ is a constant vector.

Next, we shall seek appropriate $E_{1}, E_{2}$ in (65) (67), respectively, by the definition of $A$ and (60), we have

$$
\dot{W}_{20}(\theta)=\int_{-1}^{0} d \eta(\theta) W_{20}(\theta)=2 i \omega_{0} \tau_{k} W_{20}-H_{20}(0)
$$

and

$$
\dot{W}_{11}(\theta)=\int_{-1}^{0} d \eta(\theta) W_{11}(\theta)=-H_{11}(0),
$$

where $\eta(\theta)=\eta(\theta, 0)$. By (56), we get

$$
H_{20}(0)=-g_{20} q(0)-\bar{g}_{02} \bar{q}(0)+2 \tau_{k}\left(\begin{array}{c}
0 \\
-l \beta \\
h+k \alpha^{2}
\end{array}\right),
$$



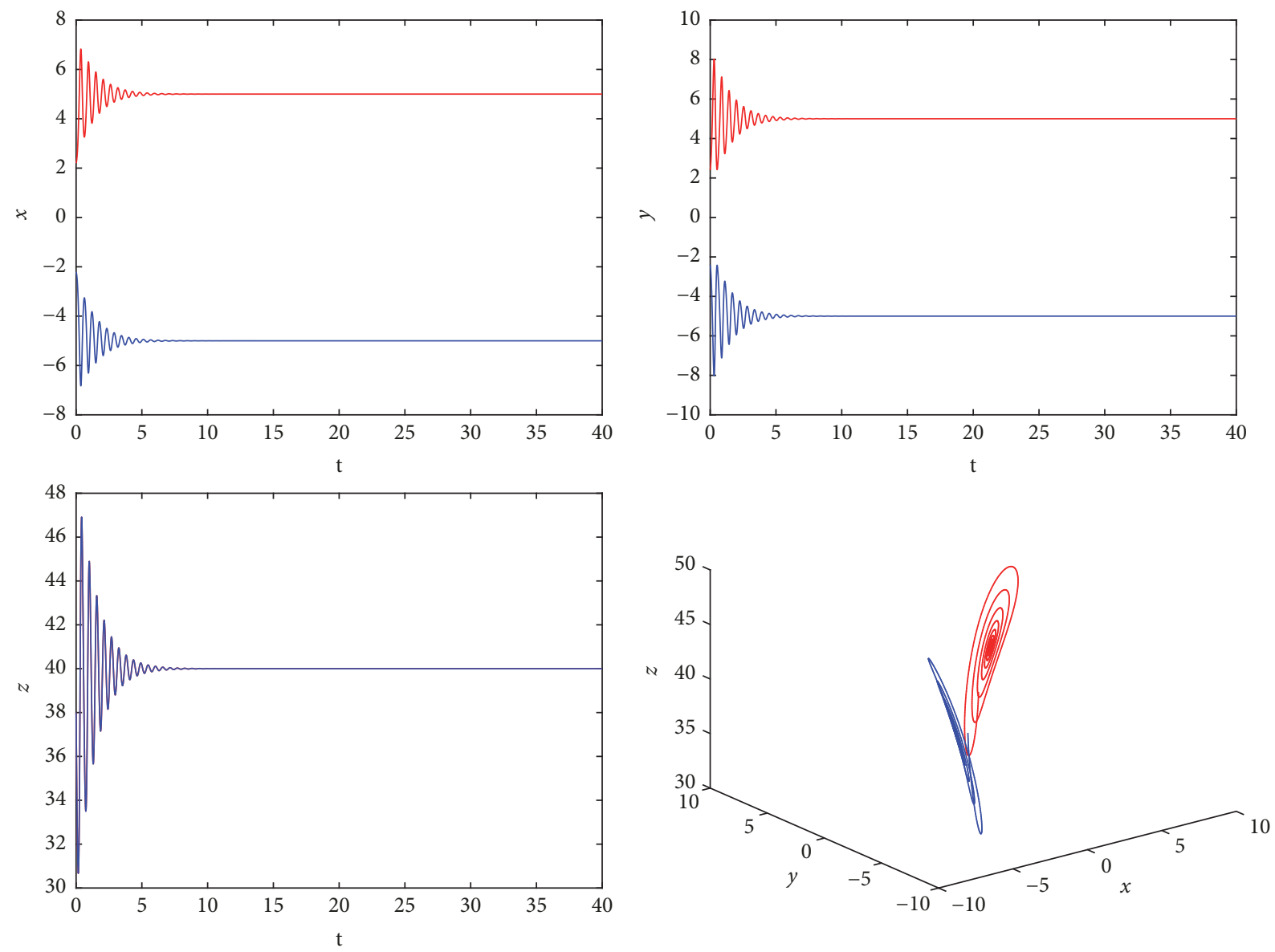

FIgURE 2: Chaos vanishes and equilibrium $E_{+}$becomes locally asymptotically stable with $\tau=0.2$.

and

$$
H_{11}(0)=-g_{11} q(0)-\bar{g}_{11} \bar{q}(0)+\tau_{k}\left(\begin{array}{c}
0 \\
-l(\beta+\bar{\beta}) \\
2 h+2 k \alpha \bar{\alpha}
\end{array}\right)
$$

For $i \omega_{k} \tau_{k}$ is the eigenvalues of $A(0)$ and $q(0)$ is the corresponding eigenvector, we obtain

$$
\begin{gathered}
\left(i \omega_{k} \tau_{k} I-\int_{-1}^{0} e^{i \theta \omega_{k} \tau_{k}} d \eta(\theta)\right) q(0)=0, \\
\left(-i \omega_{k} \tau_{k} I-\int_{-1}^{0} e^{-i \theta \omega_{k} \tau_{k}} d \eta(\theta)\right) \bar{q}(0)=0 .
\end{gathered}
$$

By substituting (65) and (68) into (70), we obtain

$$
\begin{aligned}
& \left(2 i \omega_{k} \tau_{k} I-\int_{-1}^{0} e^{2 i \theta \omega_{k} \tau_{k}} d \eta(\theta)\right) E_{1} \\
& =2 \tau_{k}\left(\begin{array}{c}
0 \\
-l \beta \\
h+k \alpha^{2}
\end{array}\right)
\end{aligned}
$$

which leads to

$$
\begin{aligned}
& \left(\begin{array}{ccc}
2 i \omega_{k}+a & -a & 0 \\
l z_{0}-b & 2 i \omega_{k}-K+K e^{-2 \omega_{k} \tau} & l x_{0} \\
-2 h x_{0} & -2 k y_{0} & 2 i \omega_{k}+c
\end{array}\right) E_{1} \\
& =2\left(\begin{array}{c}
0 \\
-l \beta \\
h+k \alpha^{2}
\end{array}\right) .
\end{aligned}
$$

It follows that

$$
\begin{aligned}
& E_{1}^{(1)}=\frac{\Delta_{11}}{\Delta_{1}}, \\
& E_{1}^{(2)}=\frac{\Delta_{12}}{\Delta_{1}}, \\
& E_{1}^{(3)}=\frac{\Delta_{13}}{\Delta_{1}},
\end{aligned}
$$



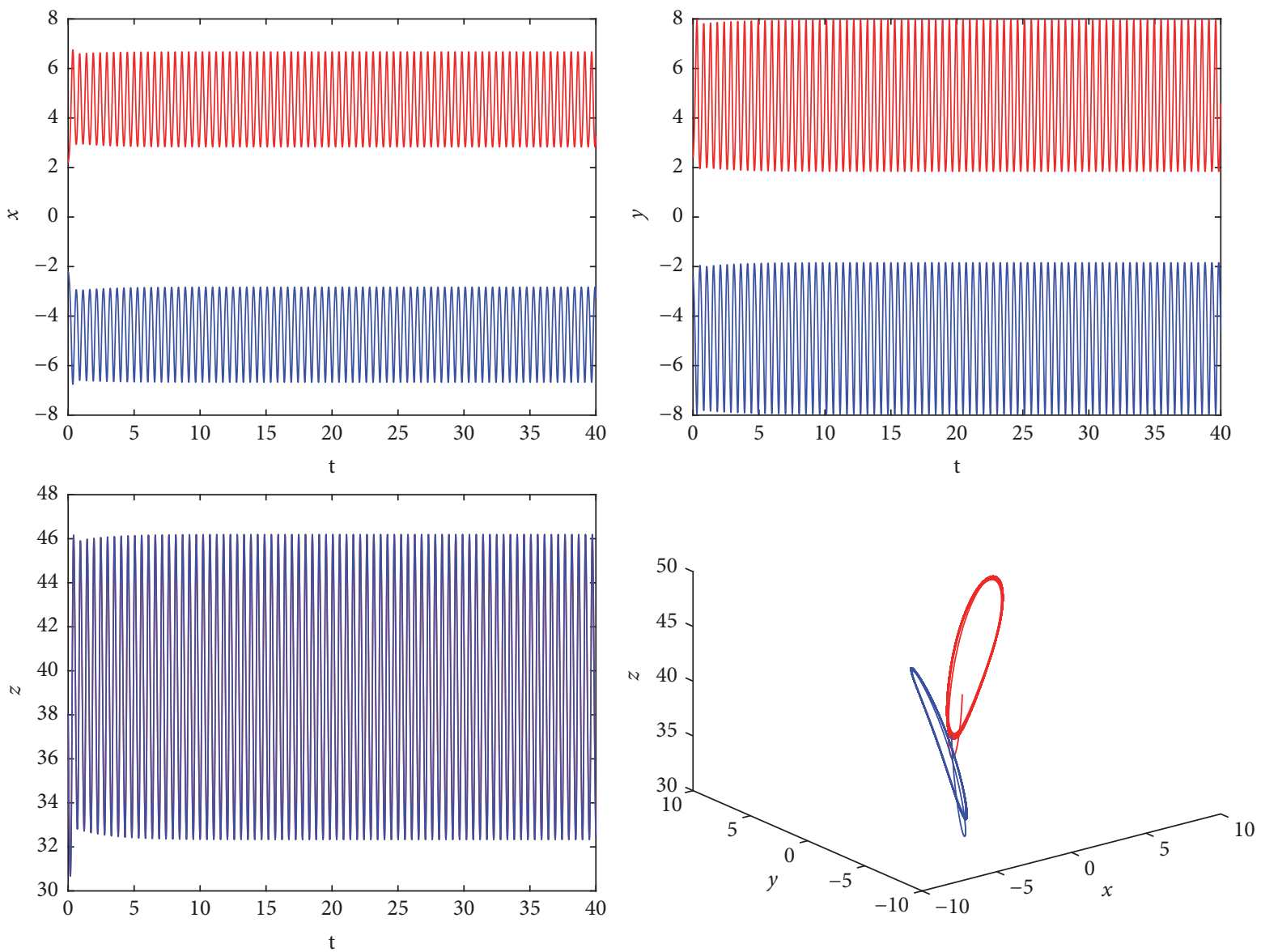

FIgURE 3: Chaos vanishes and periodic solution bifurcates from the equilibrium $E_{+}$with $\tau=0.4$.

where

$$
\begin{aligned}
& \Delta_{11}=2\left|\begin{array}{ccc}
0 & -a & 0 \\
-l \beta & 2 i \omega_{k}-K+K e^{-2 \omega_{k} \tau} & l x_{0} \\
h+k \alpha^{2} & -2 k y_{0} & 2 i \omega_{k}+c
\end{array}\right|, \\
& \Delta_{12}=2\left|\begin{array}{ccc}
2 i \omega_{k}+a & 0 & 0 \\
l z_{0}-b & -l \beta & l x_{0} \\
-2 h x_{0} & h+k \alpha^{2} & 2 i \omega_{k}+c
\end{array}\right|, \\
& \Delta_{13}=2\left|\begin{array}{ccc}
2 i \omega_{k}+a & -a \\
l z_{0}-b & 2 i \omega_{k}-K+K e^{-2 \omega_{k} \tau} & -l \beta \\
-2 h x_{0} & -2 k y_{0} & h+k \alpha^{2}
\end{array}\right|, \\
& \Delta_{1}=\left|\begin{array}{ccc}
2 i \omega_{k}+a & -a & 0 \\
l z_{0}-b & 2 i \omega_{k}-K+K e^{-2 \omega_{k} \tau} & l x_{0} \\
-2 h x_{0} & -2 k y_{0} & 2 i \omega_{k}+c
\end{array}\right| .
\end{aligned}
$$

Similarly, by substituting (67) and (71) into (69), we have

$$
\left(\begin{array}{ccc}
a & -a & 0 \\
l z_{0}-b & 0 & l x_{0} \\
-2 h x_{0} & -2 k y_{0} & c
\end{array}\right) E_{2}=\left(\begin{array}{c}
0 \\
-l(\beta+\bar{\beta}) \\
2 h+2 k \alpha \bar{\alpha}
\end{array}\right)
$$

and, hence,

$$
\begin{aligned}
& E_{2}^{(1)}=\frac{\Delta_{21}}{\Delta_{2}}, \\
& E_{2}^{(2)}=\frac{\Delta_{22}}{\Delta_{2}}, \\
& E_{2}^{(3)}=\frac{\Delta_{23}}{\Delta_{2}},
\end{aligned}
$$

where

$$
\begin{aligned}
& \Delta_{21}=\left|\begin{array}{ccc}
0 & -a & 0 \\
-l(\beta+\bar{\beta}) & 0 & l x_{0} \\
2 h+2 k \alpha \bar{\alpha} & -2 k y_{0} & c
\end{array}\right|, \\
& \Delta_{22}=\left|\begin{array}{ccc}
a & 0 & 0 \\
l z_{0}-b & -l(\beta+\bar{\beta}) & l x_{0} \\
-2 h x_{0} & 2 h+2 k \alpha \bar{\alpha} & c
\end{array}\right|, \\
& \Delta_{23}=\left|\begin{array}{ccc}
a & -a & 0 \\
l z_{0}-b & 0 & -l(\beta+\bar{\beta}) \\
-2 h x_{0} & -2 k y_{0} & 2 h+2 k \alpha \bar{\alpha}
\end{array}\right|,
\end{aligned}
$$



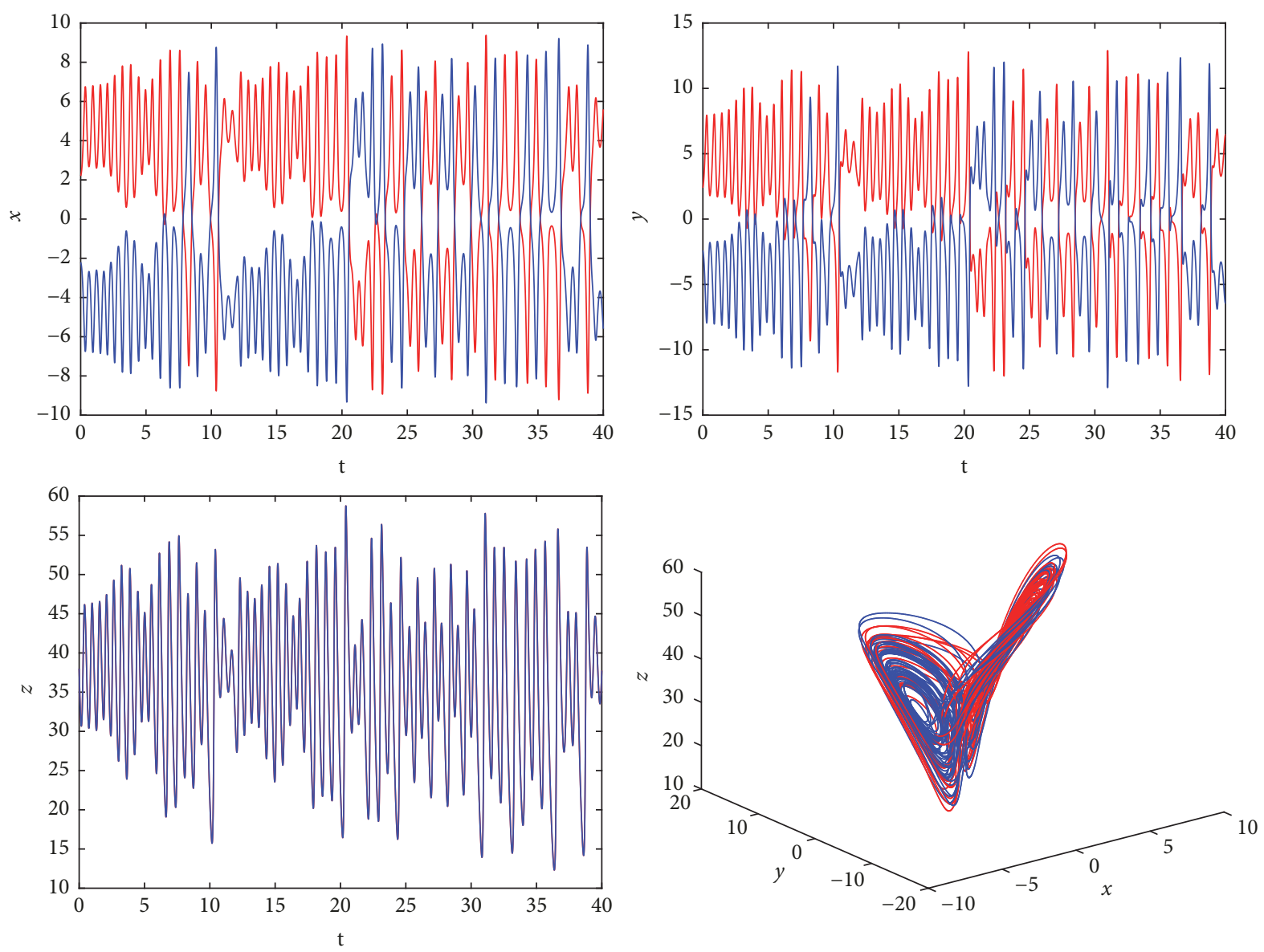

FIGURE 4: Chaos appears again with $\tau=0.62$.

$$
\Delta_{2}=\left|\begin{array}{ccc}
a & -a & 0 \\
l z_{0}-b & 0 & l x_{0} \\
-2 h x_{0} & -2 k y_{0} & c
\end{array}\right| .
$$

Consequently, we can determine $W_{20}(0)$ and $W_{11}(0)$ by (65) and (67). Furthermore, using them we can compute $g_{21}$. Therefore, all $g_{i j}$ are determined by the parameters and delay in (29). After that, we can easily compute the following values:

$$
\begin{aligned}
C_{1}(0) & =\frac{i}{2 \omega_{k} \tau_{k}}\left(g_{11} g_{20}-2\left|g_{11}\right|^{2}-\frac{1}{3}\left|g_{02}\right|^{2}\right)+\frac{g_{21}}{2}, \\
\mu_{2} & =-\frac{\operatorname{Re}\left\{C_{1}(0)\right\}}{\operatorname{Re}\left\{\lambda^{\prime}\left(\tau_{k}\right)\right\}} \\
\beta_{2} & =2 \operatorname{Re}\left\{C_{1}(0)\right\} \\
T_{2} & =-\frac{\operatorname{Im}\left\{C_{1}(0)\right\}+\mu_{2} \operatorname{Im}\left\{\lambda^{\prime}\left(\tau_{k}\right)\right\}}{\omega_{k} \tau_{k}} .
\end{aligned}
$$

Thus, we have the main results of this section.
Theorem 6. For (80), we have the following:

(1) The sign of $\mu_{2}$ determines the direction of the Hopf bifurcation; if $\mu_{2}>0\left(\mu_{2}<0\right)$, then the Hopf bifurcation is supercritical (subcritical) and the bifurcating period solutions exist for $\tau>\tau_{k}\left(\tau<\tau_{k}\right)$;

(2) The sign of $\beta_{2}$ determines the stability of the bifurcating period solutions; if $\beta_{2}<0\left(\beta_{2}>0\right)$, then the bifurcation period solutions are orbitally stable (unstable);

(3) The sign of $T_{2}$ determines the period of the bifurcating periodic solutions; if $T_{2}>0\left(T_{2}<0\right)$, then the period increases (decreases).

\section{Application to Control of Chaos}

In this section, we perform some numerical simulations to verify the results of theoretical analysis in the previous section. Now we consider the following system:

$$
\begin{aligned}
& \dot{x}(t)=10(y(t)-x(t)), \\
& \dot{y}(t)=40 x(t)-1 x(t) z(t)+K(y(t)-y(t-\tau)), \\
& \dot{z}(t)=-2.5 z(t)+2 x^{2}(t)+2 y^{2}(t),
\end{aligned}
$$

which has three equilibrium points $E_{0}(0,0,0), E_{+}(5,5,40)$, and $E_{-}(-5,-5,40)$. 

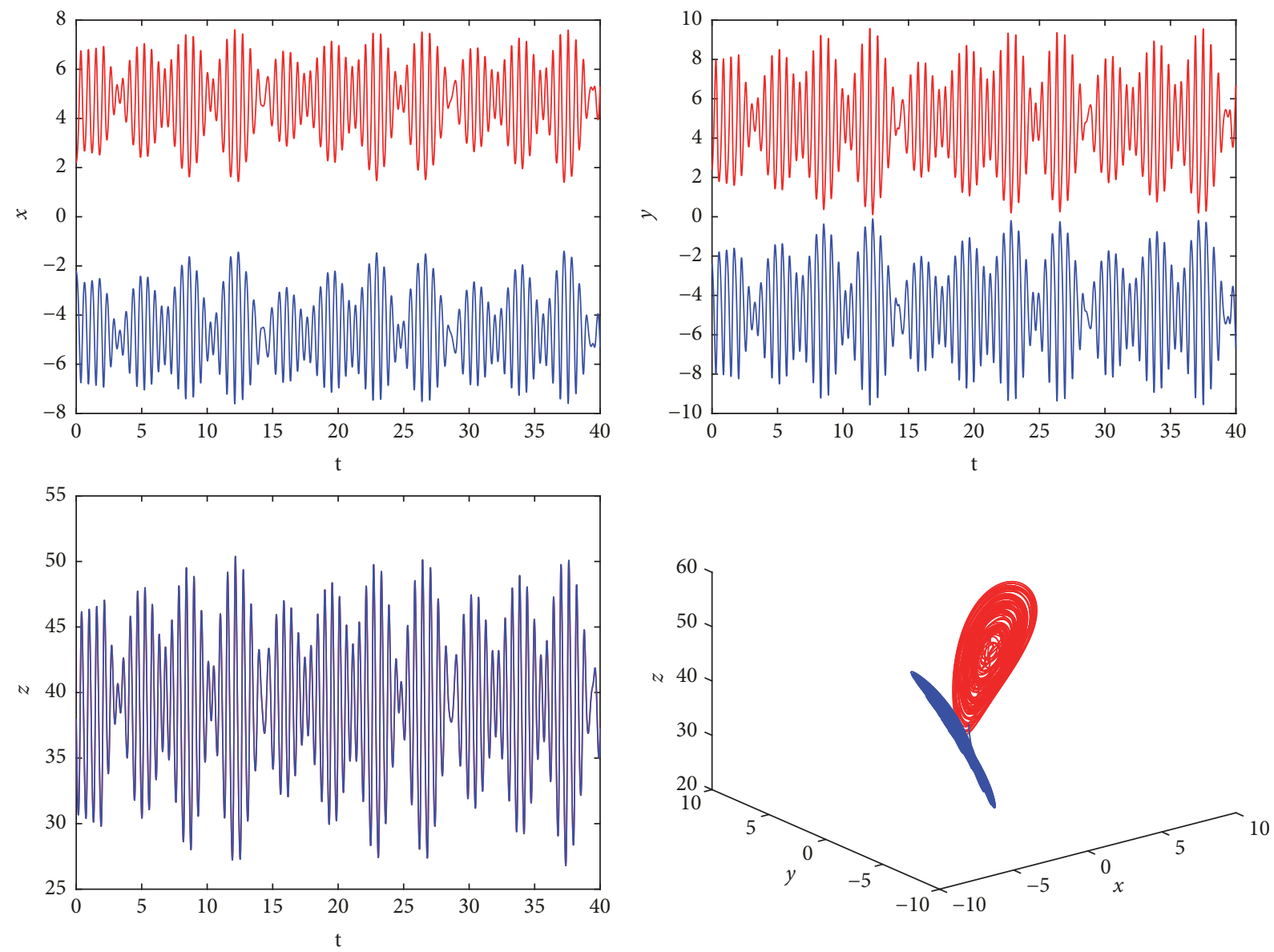

FIGURE 5: Chaos vanishes and periodic solution bifurcates from the equilibrium $E_{+}$with $\tau=2$.

For the equilibrium point $E_{0}(0,0,0)$, the characteristic equation of system (81) will be

$$
(\lambda+10)(\lambda+2.5)\left(\lambda-K+K e^{-\lambda \tau}\right)=0
$$

It is clear that (82) will be have a positive root and two negative roots. By Lemma 2 and $\Delta>0$ for all $K \in R$ it is clear that (82) has at least one root with positive real part for $K \in R$ which means that $E_{0}(0,0,0)$ is always unstable. Furthermore, we conclude that the bifurcating periodic solution is also unstable in the phase space even though they are stable in the center manifold.

For the equilibria $E_{1}(5,5,40)$ and $E_{2}(-5,-5,40)$, the characteristic equation of system (81) will be

$$
\begin{aligned}
\lambda^{3}+ & (12.5-K) \lambda^{2}+(125-12.5 K) \lambda+2000-25 K \\
& +\left(\lambda^{2}+12.5 \lambda+25\right) K e^{-\lambda \tau}=0 .
\end{aligned}
$$

Clearly, when $\tau=0$ (83) has a negative root and a pair of complex roots with positive real parts. Following Section 2, we can obtain $p=-93.75, q=-34375+1500 K$, and $r=$ $-10000(K-40) \Delta=-4500(K-24.8697)$, for all $K \in R$. When $40>K>24.8697, r>0, \Delta<0$. Thus we know that (82) has two roots with positive real parts for all $\tau \geqslant 0$, and the equilibrium points $E_{ \pm}$of system (81) is unstable. Therefore, for the purpose of control of chaos, we only consider $K<$ 24.8697. In particular, we take $K=-2$, that is,

$$
\begin{aligned}
& \dot{x}(t)=10(y(t)-x(t)), \\
& \dot{y}(t)=40 x(t)-1 x(t) z(t)-2(y(t)-y(t-\tau)), \\
& \dot{z}(t)=-2.5 z(t)+2 x^{2}(t)+2 y^{2}(t) .
\end{aligned}
$$

In this case, we can compute

$$
\begin{aligned}
z_{1} & =126.2066, \\
\omega_{1} & =11.2342, \\
\tau_{1}^{(j)} & =0.1169+\frac{2 j \pi}{\omega_{1}}, \\
h^{\prime}\left(z_{1}\right) & =-13254.4 . \\
z_{2} & =166.9167, \\
\omega_{2} & =12.9196, \\
\tau_{2}^{(j)} & =0.1070+\frac{2 j \pi}{\omega_{2}}, \\
h^{\prime}\left(z_{2}\right) & =14911.6 .
\end{aligned}
$$



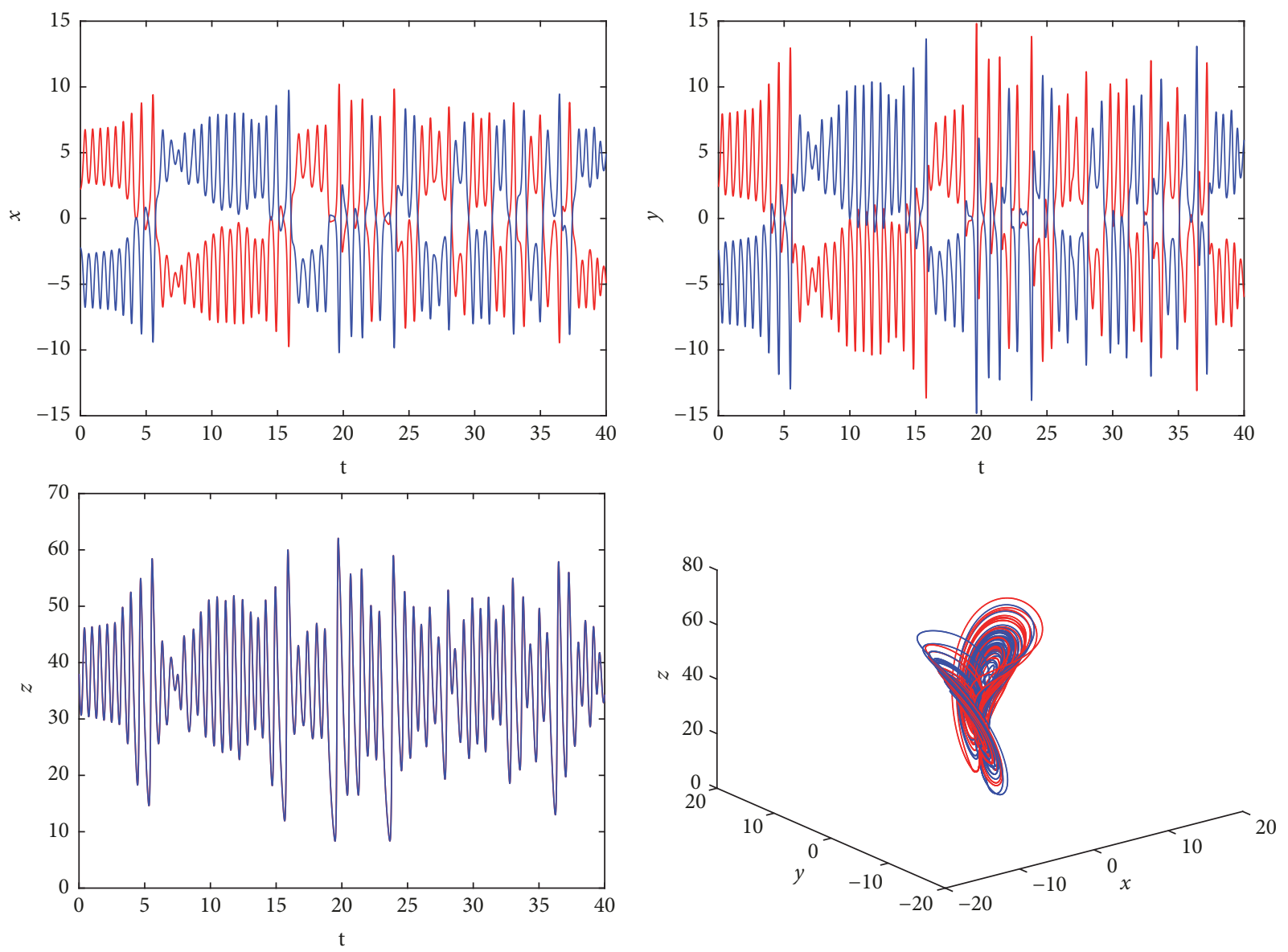

FIGURE 6: Chaos appears again with $\tau=2.97$.

In addition, notice that

$$
\begin{aligned}
\tau_{2}^{(0)} & =0.1071<\tau_{1}^{(0)}=0.1169<\tau_{2}^{(1)}=0.5935<\tau_{1}^{(1)} \\
& =0.6762 \cdots .
\end{aligned}
$$

From the arithmetic in Section 3, when $\tau=\tau_{2}^{(0)}$, we can compute $C_{1}(0)=0.0421-0.1945 i$. Thus the equilibrium point $E_{ \pm}$is asymptotically stable as is illustrated in Figure 2. When $\tau$ passes through the critical values $\tau_{2}^{0}, E_{+}$loses its stability and a Hopf bifurcation occurs. Since $\mu_{2}>0$ and $\beta_{2}<0$, the Hopf bifurcation is supercritical and the direction of the Hopf bifurcation is $\tau>\tau_{2}^{0}$ and these bifurcating period solutions from $E_{ \pm}$are stable; see Figures 3 and 5. On the other hand, the numerical simulations show that the bifurcating periodic solutions disappear when the delay $\tau_{1}^{0}>\tau>\tau_{2}^{0}$ and chaos occurs again; see Figures 4 and 6.

\section{Conclusions}

In this paper, we aim to investigate delay feedback control of a new butterfly-shaped Lorenz-like system. Taking the time delay as a bifurcation parameter, we explore the effect of time delay on its dynamics. The local stability and the existence of Hopf bifurcation of the chaotic system is investigated by analyzing the characteristic equations. After that, we also investigate the direction of the Hopf bifurcation and the stability of the bifurcating periodic solutions, which are determined by applying the normal form theory and the center manifold theorem. Our theoretical results and numerical simulations show that when $\tau$ passes through some certain critical values, the stability of the system will change from unstable to stable, or the stable periodic orbit will be branched out from the equilibrium, which will lead to the chaos phenomenon in the system disappear, and achieve the purpose of controlling chaos.

\section{Data Availability}

The data used to support the findings of this study are available from the corresponding author upon request.

\section{Conflicts of Interest}

The authors declare that there are no conflicts of interest regarding the publication of this paper.

\section{Acknowledgments}

This work was supported by the National Natural Science Foundation of China (Grant no. 61761002) and the 
Graduate Innovation Project of North Minzu University (no. YCX18091) and the First-Class Disciplines Foundation of Ningxia (Grant no. NXYLXK2017B09).

\section{References}

[1] O. E. Rössler, "An equation for hyperchaos," Physics Letters A, vol. 71, no. 2-3, pp. 155-157, 1979.

[2] T. S. Parker and L. O. Chua, "Chaos: A Tutorial for Engineers," Proceedings of the IEEE, vol. 75, no. 8, pp. 982-1008, 1987.

[3] G. Chen and X. Dong, From Chaos to Order: Methodologies, Perspectives and Applications, vol. 13, World Scientific, Singapore, 1998.

[4] Z. Jiang and W. Ma, "Delayed feedback control and bifurcation analysis in a chaotic chemostat system," International Journal of Bifurcation and Chaos, vol. 25, no. 6, 2015.

[5] J. Yang, E. Zhang, and M. Liu, "Bifurcation Analysis and Chaos Control in a Modified Finance System with Delayed Feedback," International Journal of Bifurcation \& Chaos, vol. 26, no. 6, Article ID 1650105, 2016.

[6] X.-Y. Meng, H.-F. Huo, X.-B. Zhang, and H. Xiang, "Stability and Hopf bifurcation in a three-species system with feedback delays," Nonlinear Dynamics, vol. 64, no. 4, pp. 349-364, 2011.

[7] A. Buscarino, L. Fortuna, M. Frasca, and G. Sciuto, "Design of time-delay chaotic electronic circuits," IEEE Transactions on Circuits and Systems I: Regular Papers, vol. 58, no. 8, pp. 18881896, 2011.

[8] A. Buscarino, L. Fortuna, M. Frasca, L. V. Gambuzza, and G. Sciuto, "Memristive chaotic circuits based on cellular nonlinear networks," International Journal of Bifurcation and Chaos, vol. 22, no. 3, 2012.

[9] Z. Wei, V.-T. Pham, T. Kapitaniak, and Z. Wang, "Bifurcation analysis and circuit realization for multiple-delayed WangChen system with hidden chaotic attractors," Nonlinear Dynamics, vol. 85, no. 3, pp. 1635-1650, 2016.

[10] E. Ott, C. Grebogi, and J. A. Yorke, "Controlling chaos," Physical Review Letters, vol. 64, no. 11, pp. 1196-1199, 1990.

[11] K. Pyragas, "Continuous control of chaos by self-controlling feedback," Physics Letters A, vol. 170, no. 6, pp. 421-428, 1992.

[12] K. Pyragas, "Control of chaos via extended delay feedback," Physics Letters A, vol. 206, no. 5-6, pp. 323-330, 1995.

[13] L. Chongxin, L. Ling, L. Tao, and L. Peng, "A new butterflyshaped attractor of Lorenz-like system," Chaos, Solitons \& Fractals, vol. 28, no. 5, pp. 1196-1203, 2006.

[14] R. Wu and T. Fang, "Stability and Hopf bifurcation of a Lorenzlike system," Applied Mathematics and Computation, vol. 262, pp. 335-343, 2015.

[15] G. Brown, C. M. Postlethwaite, and M. Silber, “Time-delayed feedback control of unstable periodic orbits near a subcritical Hopf bifurcation," Physica D: Nonlinear Phenomena, vol. 240, no. 9-10, pp. 859-871, 2011.

[16] V. Pyragas and K. Pyragas, "Delayed feedback control of the Lorenz system: an analytical treatment at a subcritical Hopf bifurcation," Physical Review E: Statistical, Nonlinear, and Soft Matter Physics, vol. 73, no. 3, 036215, 10 pages, 2006.

[17] J. Guan and S. Qin, "Distributed delay feedback control of a new butterfly-shaped chaotic system," Optik - International Journal for Light and Electron Optics, vol. 127, no. 14, pp. 5552-5561, 2016.

[18] X. F. Wang, G. Chen, and X. Yu, "Anticontrol of chaos in continuous-time systems via time-delay feedback," Chaos: An
Interdisciplinary Journal of Nonlinear Science, vol. 10, no. 4, pp. 771-779, 2000.

[19] Y. Feng and Z. Wei, "Delayed feedback control and bifurcation analysis of the generalized Sprott B system with hidden attractors," European Physical Journal Special Topics, vol. 224, no. 8, pp. 1619-1636, 2015.

[20] G. Kociuba and N. R. Heckenberg, "Controlling chaos in a Lorenz-like system using feedback," Physical Review E: Statistical, Nonlinear, and Soft Matter Physics, vol. 68, no. 6, pp. 662121662128, 2003.

[21] Y. Song and J. Wei, "Bifurcation analysis for Chens system with delayed feedback and its application to control of chaos," Chaos Solitons \& Fractals, vol. 22, no. 1, pp. 75-91, 2004.

[22] Q.-L. Han, "On designing time-varying delay feedback controllers for master-slave synchronization of Lur'e systems," IEEE Transactions on Circuits and Systems I: Regular Papers, vol. 54, no. 7, pp. 1573-1583, 2007.

[23] R. Zhang, "Bifurcation analysis for $\mathrm{T}$ system with delayed feedback and its application to control of chaos," Nonlinear Dynamics, vol. 72, no. 3, pp. 629-641, 2013.

[24] J. K. Hale, Theory of Functional Differential Equations, Springer, New York, NY, USA, 1977.

[25] B. D. Hassard, N. D. Kazarinoff, and Y.-H. Wan, Theory and Applications of Hopf Bifurcation, Cambridge University Press, 1981.

[26] S. Ruan and J. Wei, "On the zeros of a third degree exponential polynomial with applications to a delayed model for the control of testosterone secretion," Ima Journal of Mathematics Applied in Medicine \& Biology, vol. 18, no. 1, 41 pages, 2001.

[27] S. G. Ruan and J. J. Wei, "On the zeros of transcendental functions with applications to stability of delay differential equations with two delays," Dynamics of Continuous, Discrete \& Impulsive Systems A: Mathematical Analysis, vol. 10, no. 6, pp. 863-874, 2003. 


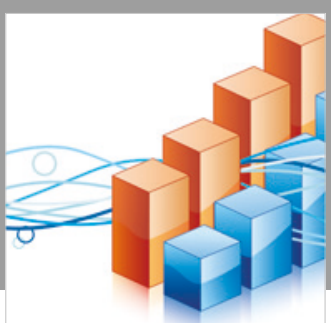

Advances in

Operations Research

\section{-n-m}
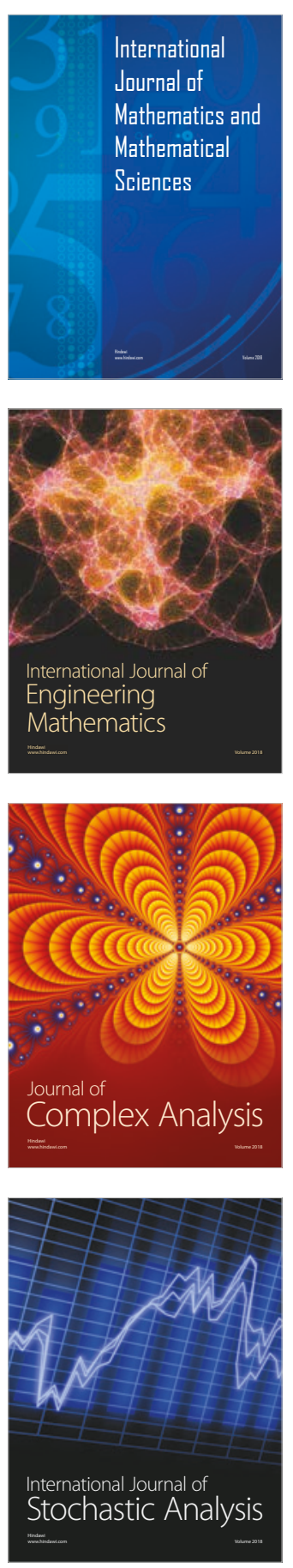
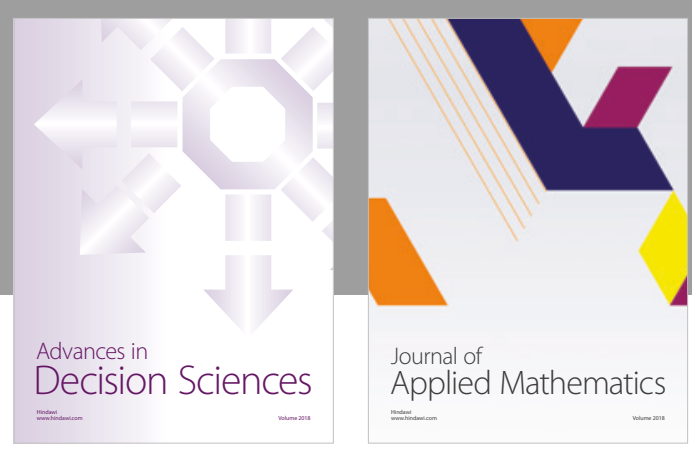

Journal of

Applied Mathematics
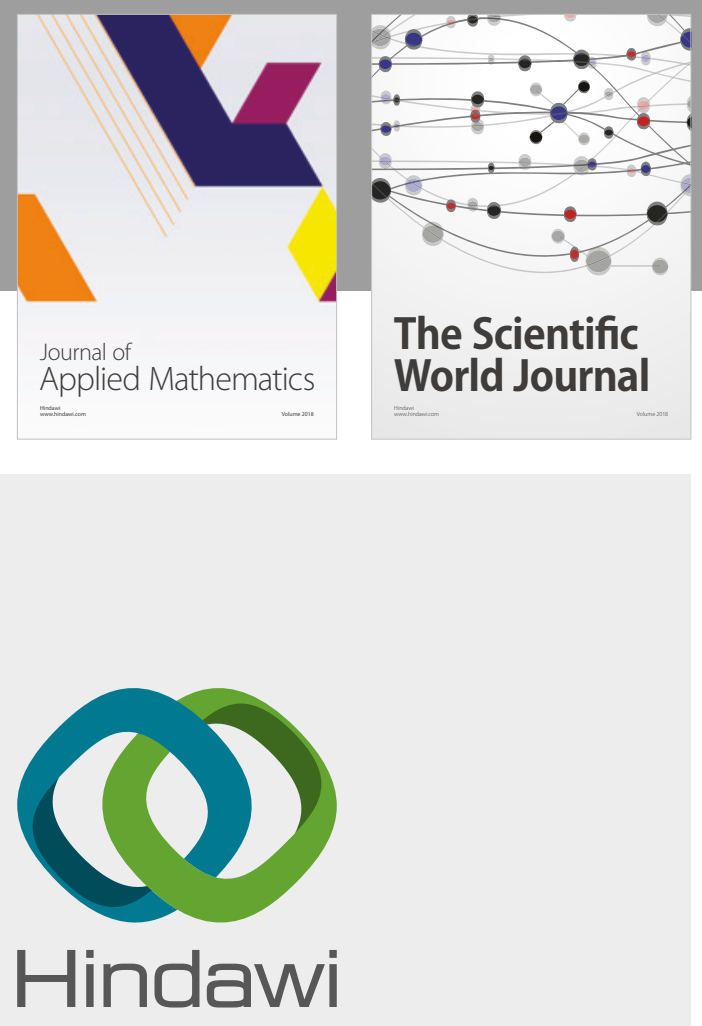

Submit your manuscripts at

www.hindawi.com

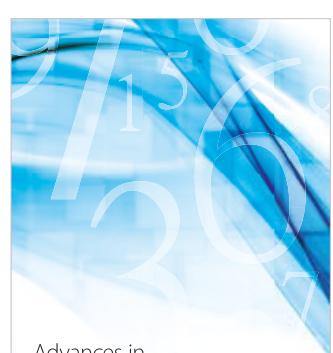

Advances in
Numerical Analysis
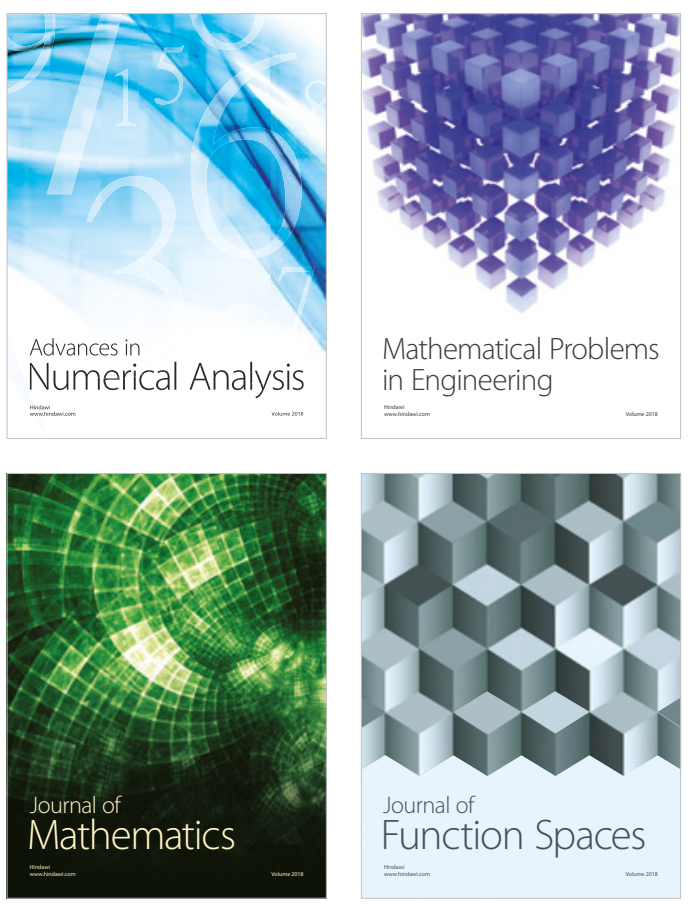

Mathematical Problems in Engineering

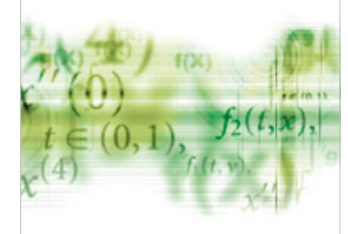

International Journal of

Differential Equations

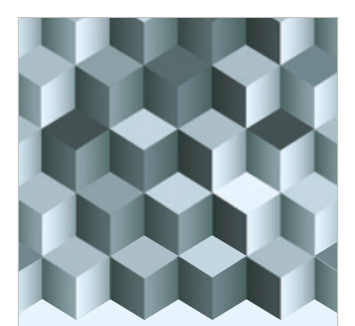

Journal of

Function Spaces

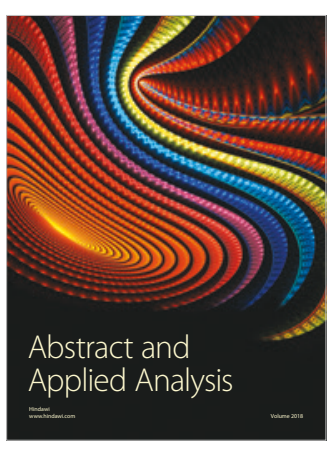

The Scientific

World Journal

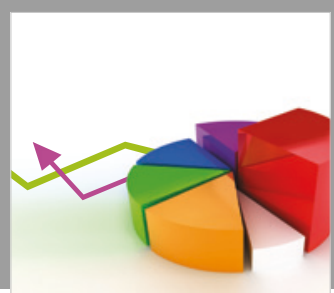

Journal of

Probability and Statistics
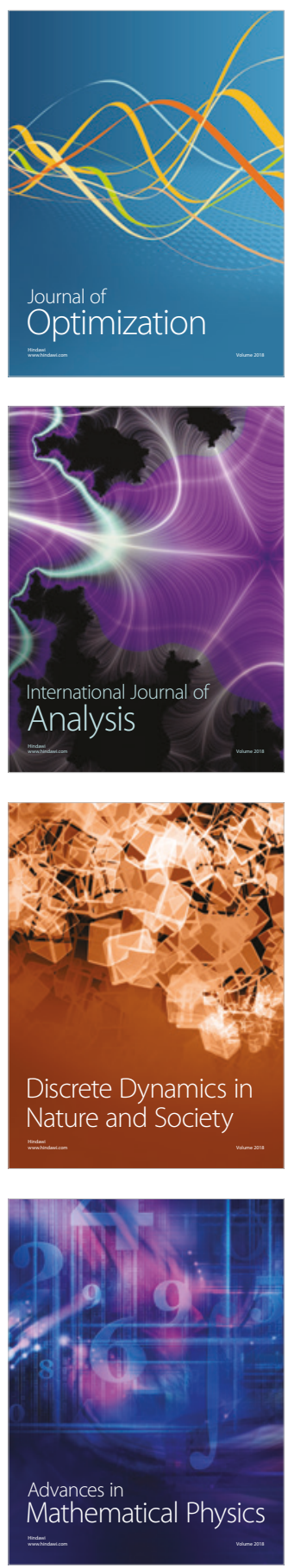